\title{
Transient Analysis of Queueing Model
}

\author{
Shyam Sundar Sah*, Ram Prasad Ghimire \\ Department of Mathematical Sciences, Kathmandu University, Nepal \\ *corresponding author: writeme.shyam@gmail.com
}

\begin{abstract}
This paper deals with the study of Erlangian queueing system with time dependent framework. Under our study we find (i) the expected number of customers in the queue (ii) the expected waiting time before being served (iii) the expected time spent in the system (iv) the expected number of customers in the system. Customers arrive in the system in Poisson fashion with rate and served in arbitrary service time distribution with rate $\mu$.The probability generating function technique and Laplace transform method have been used. The numerical computation has also been obtained for applicability of the model.
\end{abstract}

Keywords: Erlangian, Laplace probability, Poisson, Generating function

\section{Introduction}

Transient analysis is dependent on time, it uses different analysis algorithms, control options with different convergence related issues and different initialization parameters. There are tremendous real life problems where in customers come in Possion process and service of customers follows phase type distribution i.e. Erlang distribution. From time to time Erlang distribution queuing models have been studied by several authors under various provisions that meet real life situation so it is worthwhile to mention some of the work done on the line. Pearn and chang [15] studied optimal management problem of the N-policy $\mathrm{M} / \mathrm{E}_{\mathrm{k}} / 1$ queuing system with removable service station under steady state condition. Griffins et al. [10] evaluated the mean waiting time in terms of a new generation of the modified Bessel function and in the queue is evaluated. Yue et al. [20] analysed an $\mathrm{M} / \mathrm{E}_{\mathrm{k}} / 1$ queuing system with balking and state dependent service. In their model customers were served with different rates depending on the number of customers in the system. If a customer on arrival found other customers in the system, it either decided to enter the queue or balk with a constant probability. They claimed that they first formulated the queuing model as quasi-birth and death (QBD) process. Then they obtained the equilibrium condition of the system by using the matrix geometric solution method. Chan et al. [7] solved transient solution of Markovian queuing networks considering boundary value method (BVMs). By applying algebraic multigrial (AMG) methods with modified restriction operator they solved the resulting system of linear equations. Kaczynski et al. [12] studied the M/M/S queue for a positive integers and gave a method for calculating the probability distribution of the number of customers an arriving customers sees upon arrival to an $\mathrm{M} / \mathrm{M} / \mathrm{S}$ queue and also calculated the sojourn time distribution, for a given customers in this queue with $\mathrm{k}=0$ customers initially present in the system. Monsellato [14] solved the problem of the determination of the transition functions in the explicit form. They have solved the problem assuming that the solution was representable as a Taylor series, under the initial condition that the process starts to state zero. They have also discussed the same problem for the embedded chain and obtained a recursive formula for the transient distribution. Ayyappan and 
Shyamala[4] obtained the time dependent solution and the corresponding steady state solutions and they also derived the performance measures, the mean queue size and the average waiting time explicitly.

Singla and Garg [17] obtained transient state queue length probabilities and laplace transform of the generating function of transient-state queue length probabilities numerically and compared graphically various probabilities relating the model feedback queueing system with correlated departures. Li and Cheng [14] derived the transient joint queue length distribution of customers in the system using Markov skeleton processes, and showed the minimal nonnegative solution of a backward equation. Zeng et al. [21] investigated the queue length and the average waiting time of the railway container terminal gate system, as well as the optimal number of service channels during the different time period. They have also developed $\mathrm{M} / \mathrm{Ek} / 1$ transient queuing model based on the distribution of the arrival time interval and the service time by the equally likely combinations (ELC) heuristic method. They also integrated into an optimization framework to obtain the optimal operation schemes. Finally they made analysis model validation, sensitivity testing and system optimization. Al-Seedy et al. [2] have obtained the transient solution of multiserver queue with balking and reneging which inspired Ammar [3] to use a similar technique to derive a new elegant explicit solution for a two heterogeneous servers queue with impatient behavior. In addition, steady-state probabilities of the system size were studied and some important performance measures discussed for the considered system. Al- Seedy [1] obtained the transient solution of the non truncated queue: $\mathrm{M} / \mathrm{M} / 2$ with an additional server for longer queues considering the balking concept, heterogeneity and different probability in choosing the server. Chan et al. [6] applied boundary value methods to find transient solutions of $\mathrm{M} / \mathrm{M} / 2$ queueing systems with two heterogeneous servers under a variant vacation policy. They employed an iterative method to solve the resulting large linear system and used a Crank \{Nicolson pre-conditioner to accelerate the convergence $\}$ and at last presented numerical results to demonstrate the efficiency of the proposed method. Radhika and Deepika [16] solved the system of equations together with the initial conditions for an analytical expression for Pns' in each interval by considering a transient queuing system and its solution using the state space approach. For this, they sub divided the given interval of study $[0 ; \mathrm{T}]$ into small sub intervals, where it was reasonable to assume that the instantaneous arrival and service rates were constants. The solution obtained at the right end point of the previous sub interval was taken as the initial condition in the sub sequent interval. They employed the method for an (M/M/1): (FCFS/m/1) system.

Czachorski et al. [9] gave the use of the diffusion approximation in transient analysis of queueing models applied to investigate some aspects of Internet transmissions. In classical queuing theory, the analysis of transient states was complex and practically did not go far beyond MIMII queue and its modifications. However, they focused the time dependent ows in computer networks and especially in Internet on transient-state analysis, which was necessary to investigate the dynamics of TCP ows cooperating with active queue management or to see the changes of priority queues which assure the differentiated QoS. With the use of GIGIIIN and GIGIIINIPRIOR models, they presented the potentials of the diffusion approximation and compared it with alternative methods. Markovian queues solved numerically, fluid flow approximation and simulation. Vaderna and Elteto [18] investigated a queuing system with infinite number of servers where the arrival process was given by a Markov arrival process (MAP) and the service time followed a Phase-type (PH) distribution. They revealed that highly correlated arrival processes and heavy-tailed service time distributions could be approximated by these tools on a wide range of time-scales. Under the study of transient behavior of the system they analysed the time-dependent moments of the queue 
length explicitly by solving a set of differential equations. Bura and Kumar [5] obtained the timedependent solution of a varying catastrophic intensity cumrestorative Markovian queueing model with infinite capacity. The transient solution has been obtained recursively. The simulation of the model has also been performed and various measures of performance have been computed. The steady-state solution is also derived. Further, some particular cases of the queuing model have been derived and discussed. Yang and Liu [19] developed a statistical methodology, integrated with extensive offline simulation for the estimation of a small number of transfer function models (TFMs) that quantify the input-output dynamics of a general queueing system.

In this paper we study the $\mathrm{M} / \mathrm{E}_{\mathrm{k}} / 1$ queueing system with finite capacity. Our model is close to the model studied by Gupta [11]. We have made the provision of time dependent arrival and service rates functions. Customers arrive in the system with rate $\lambda(t)$ in Poisson process and are served exponentially with rate $\mu(\mathrm{t})$ in $\mathrm{K}$ - phases. We obtain the formulas for various performance measures such as (i)the expected number of customers in the queue(ii)the expected waiting time before being served (iii)the expected time spent in the system (iv)the expected number of customers in the system.

\section{Mathematical Model}

For our model we use the following notations:

$\lambda(\mathrm{t})=\mathrm{t}($ time dependent arrival rate)

$\boldsymbol{\mu}(\mathrm{t})=\mathrm{t}$ (time dependent service rate)

$\mathrm{N}=$ System capacity, $\mathrm{K}=$ Number of phase

$\mathrm{i}=$ General state

$\mathrm{z}=$ Complex parameter where $|\mathrm{z}|<1$

$\mathrm{s}=$ Laplace transform variable

$\mathrm{P}_{\mathrm{i}}(\mathrm{t})=$ Probability that there are $\mathrm{i}$ customers in any time $\mathrm{t}$
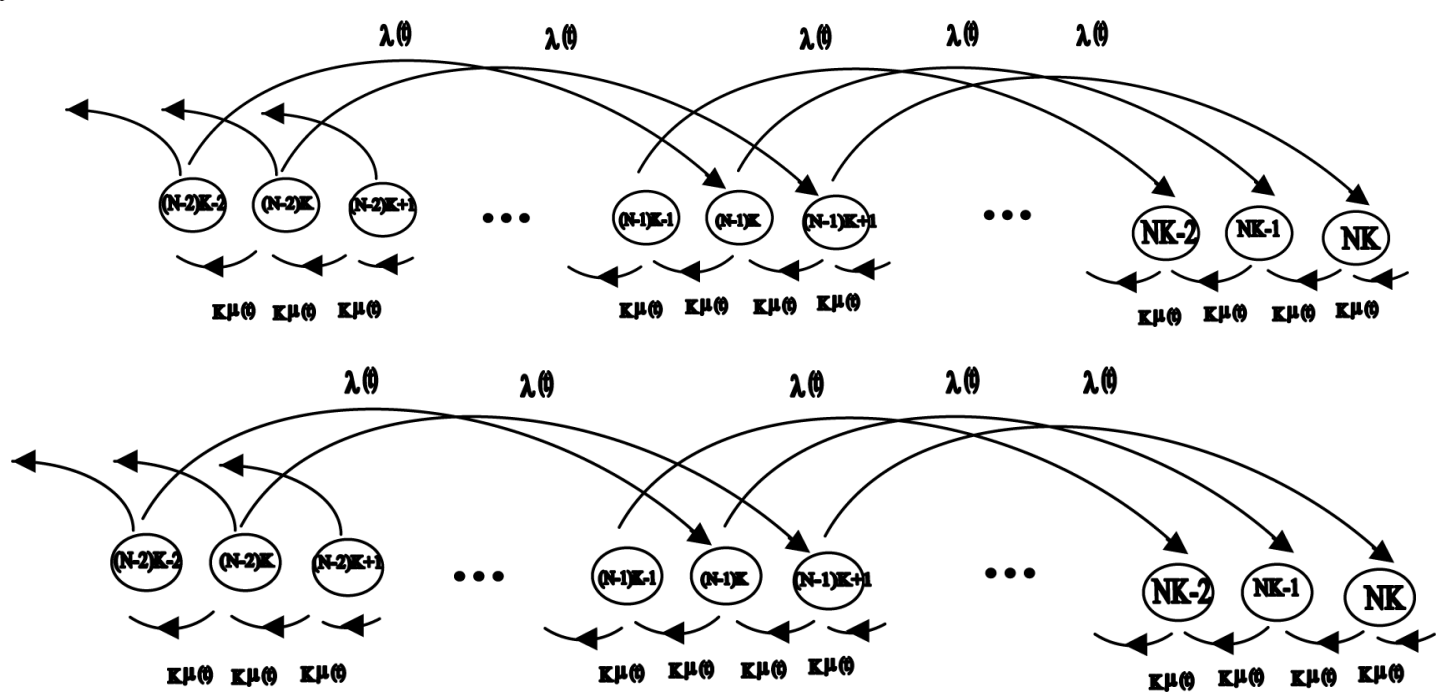

Fig 1: State Transition Diagram

For our model the differential difference equations [11] with the help of figure1 are: 


$$
\begin{aligned}
P_{o}^{\prime}(t)= & -\lambda(t) P_{0}(t)+K \mu(t) P_{1}(t) ; i=0 \\
P_{i}^{\prime}(t)=- & {[\lambda(t)+K \mu(t)] P_{i}(t)+K \mu(t) P_{i+1}(t) ; 1 \leq i<K } \\
P_{i}^{\prime}(t)=- & {[\lambda(t)+K \mu(t)] P_{i}(t)+\lambda(t) P_{i-k}(t)+K \mu(t) P_{i+1}(t) ; K \leq i \leq } \\
& (N-1) K \\
P_{i}^{\prime}(t)=- & {[K \mu(t)] P_{i}(t)+\lambda(t) P_{i-k}(t)+K \mu(t) P_{i+1}(t) ;(N-1) K<i<N K } \\
P_{N k}{ }^{\prime}(t)= & -[K \mu(t)] P_{N k}(t)+\lambda(t) P_{(N-1) k}(t)
\end{aligned}
$$

Above system of differential equations contains $(\mathrm{NK}+1)$ equations which we have to solve with the initial conditions $\mathrm{P}_{\mathrm{o}}(0)=1, \mathrm{P}_{\mathrm{i}}(0)=0$ for $\mathrm{i}>0$. We solve this system of ordinary differential equations by using the Runge-Kutta method of fourth order. We have plotted the graphs on the basis of ODE's and performance measures.

\section{Some performance measures}

(i) Average number of customers in the system $\operatorname{Ls}(\mathrm{t})=\sum_{i=1}^{N K}$ i. $P_{i}(t)=\sum_{i=1}^{N K} i .\left[l^{-1}\left(p^{*}(z, s)\right)\right]$

(ii) Average number of customers in the queue $\mathrm{Lq}(\mathrm{t})=\sum_{i=1}^{N K}(i-1) P_{i}(t)$

(iii) Expected time in the system of a customer $\mathrm{Ws}_{\mathrm{s}}(\mathrm{t})=\mathrm{W}_{\mathrm{q}}+1 / \mu(\mathrm{t})$

(iv) Expected waiting time in queue $\mathrm{Wq}(\mathrm{t})=\sum_{i=1}^{N K} P_{i} \frac{i-1}{\mu(t)}$

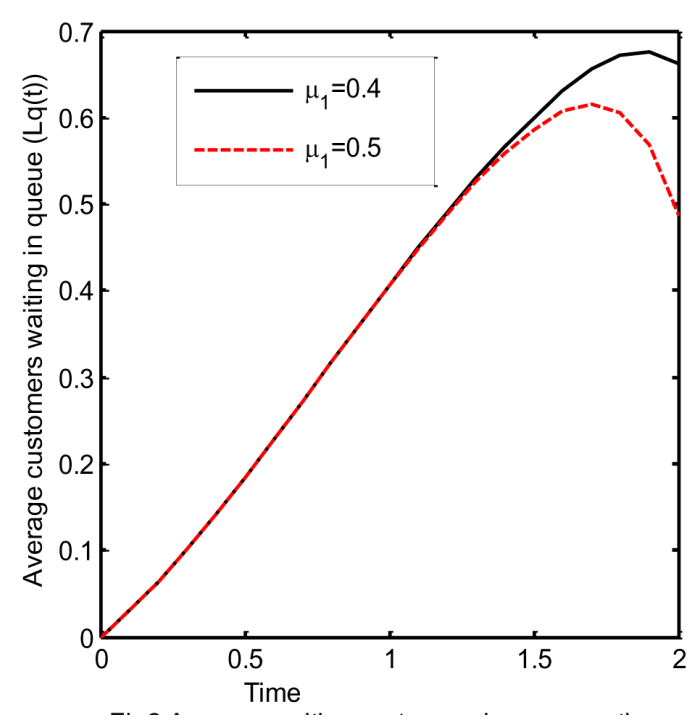

Fig. 2. Average waiting customers in queue vs time

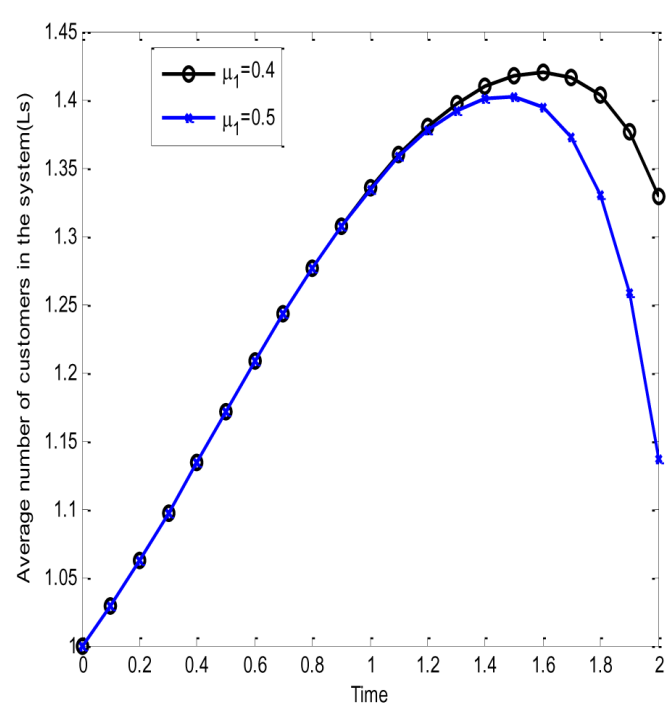

Fig. 3. Average customers in the system vs time 


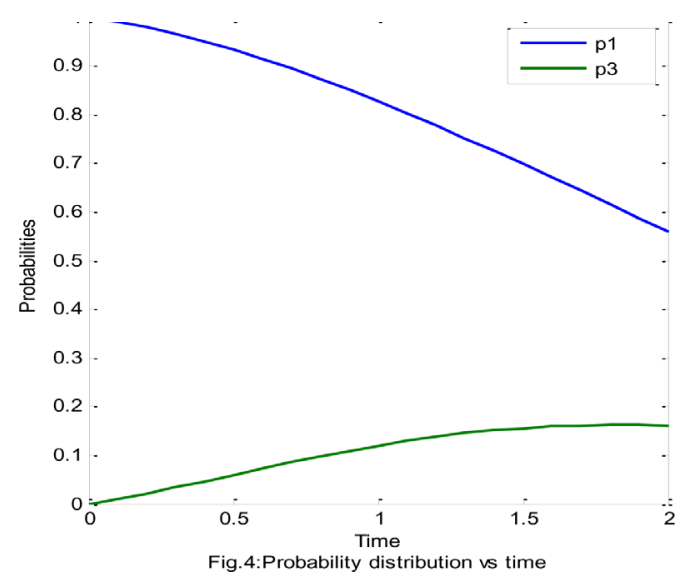

Fig. 4. Probability distribution vs time

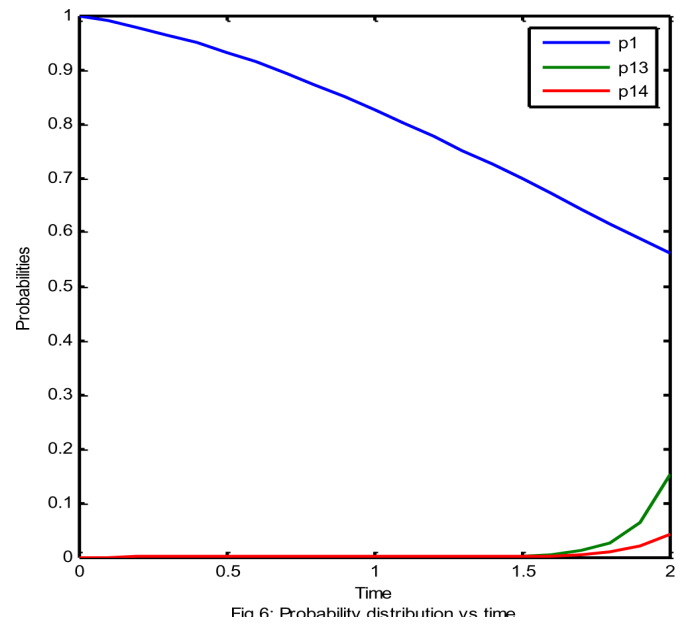

Fig. 6. Probability distribution vs time

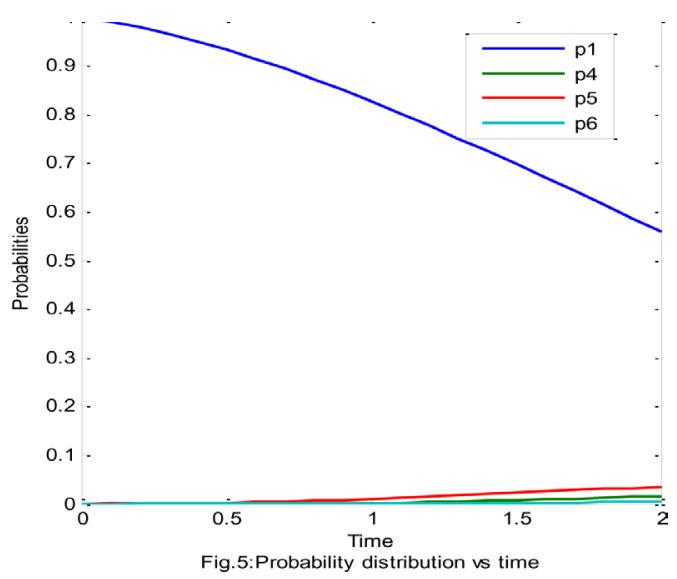

Fig. 5. Probability distribution vs time

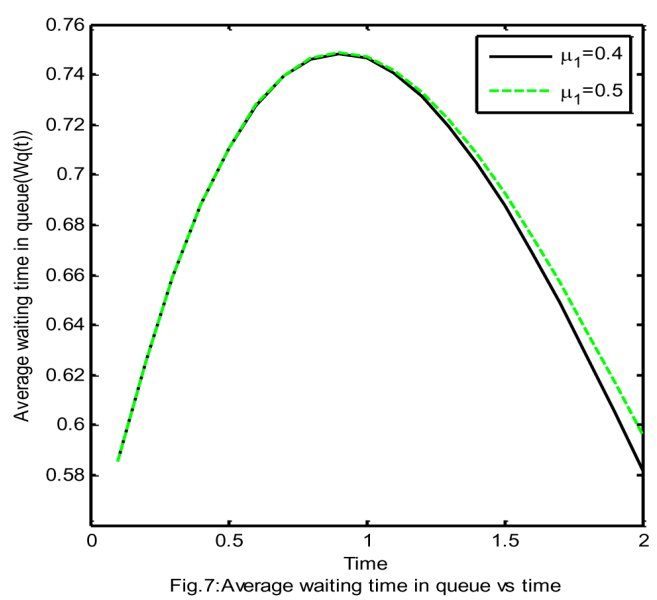

Fig. 7. Average waiting time in queue vs time

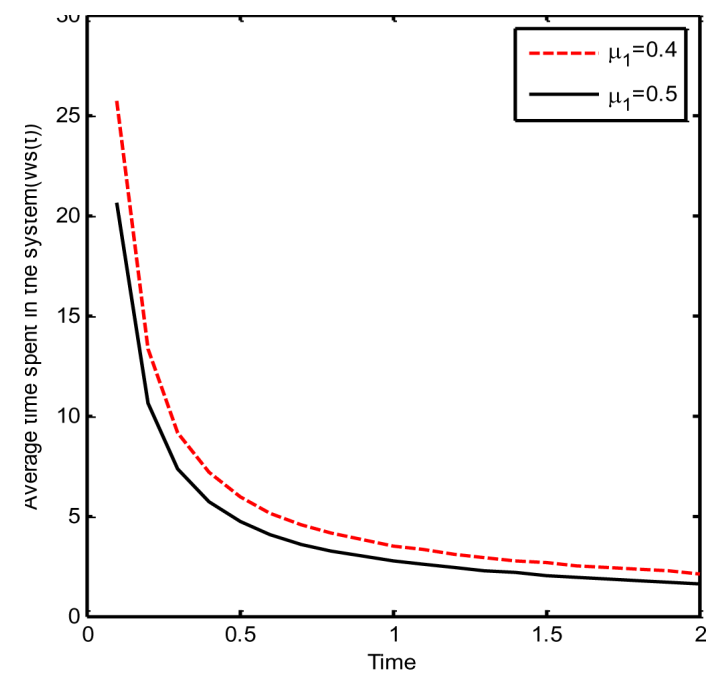

Fig. 8. Average time spent in the system vs time 


\section{Numerical Results and Discussion}

Numerical results have been obtained by using formulas (i) through (iv) by applying Runge-kutta fourth order method in the system of ordinary differential equations (1) through (5) with the help of computational software for the parametric values constant with time step under the variation of service rate as shown in the graphs. Graphs of various parameters versus time have been shown the fig. 2 through fig. 8 . Fig. 2 explores that higher the values of service rate below is the curve and smaller is the number of customers in the queue which is realistic in nature.Fig. 3 depicts that average number of customers in the system increase in the same rate for different values of service rates but after some time higher value of service rate gives lesser number of customers in the system which is quite realistic. Figs 4 through 6 explain the manner by which probabilities have been distributed over time. These figures also show that in unequillibrium conditions all probabilistic values have not been distributed equally which can be seen by the fact that initial probability has been reduced significantly due to which some the probabilities have their values zero over time which means that probabilities are not depending upon time only but upon other parameters too.Fig.7 shows that initially there is no queue but after a while queue length increases so that the waiting time increases rapidly it remains some time and decreases rapidly even at the higher service rate . Fig. 8 displays that customers arriving the system in starting time have to spend long time than the customers arriving latter on and this is more practical because our model is phase-type service time distribution model under which to complete one job, the arriving unit has to pass through various service stations which take some times to be consistent in their service formance.Fig. 8 also shows clearly that faster service rate reduces significant time to spend in the system.

\section{Conclusion}

Study of M/Ek/1 model under time dependent arrival and service rates has been made .Under the study the numerical results for various performance measures have been obtained by using RK fourth order method. This model extensively used in the traffic management system in airport where number of landing and takeoff of aircrafts can be defined by demand rate. Airport's capacity profile as the service and is defined by the service rate $\mu(t)$. Initially airport is taken to be empty but it has $\mathrm{N}$ number of aircrafts that can be occupied. This model also will be effective to reduce the congestions problem of passport department in Nepal. In the manufacturing system job arrive in Poisson fashion and before finalized of the product it has to pass through various phases. Our model can be studied under multiple servers' provision which may give more general solution under time dependent situations so as to make the model more realistic.

Acknowledgement: First author is thankful to University Grant Commission (UGC), Nepal for providing the $\mathrm{PhD}$ scholarship due to which this work has been possible.

\section{References}

[1] Al-Seedy (2004), A transient solution of the non-truncated queue $\mathrm{M} / \mathrm{M} / 2$ with balking and an additional server for longer queues (Krishnamoorthi discipline). Applied Mathematics and Computation, 156: 763-769.

[2] Al-Seedy RO, El-Sherbiny AA, El-Shehawy SA and Ammar SI ( 2009), Transient solution of the $\mathrm{M} / \mathrm{M} / \mathrm{C}$ queue with balking and reneging. Computers and Mathematics with Applications, 57: $1280-1285$.

[3] Ammar SI (2014), Transient analysis of a two heterogeneous servers queue with impatient behavior. Journal of Egyptian Mathematical Society, 22 (6):90 -95. 
[4] Ayyappan G and Shyamala S (2013), Time dependent solution of $\mathrm{m}[\mathrm{x}] / \mathrm{g} / 1$ queuing model with bernoulli vacation and balking, International Journal of Computer Applications, 61(21), 0975 - 8887.

[5] Bura GS and Kumar R (2013), Transient analysis of a limited capacity markovian queueing system subjected to varying catastrophic intensity and restoration, American Journal of Operational Research, 3(2A), 34-43.

[6] Chan RH, Lee ST, Sun HW (2012), Boundary value methods for transient solutions of queueing networks with variant vacation policy, Journal of Computational and Applied Mathematics, 236, 3948-3955.

[7] Chan RH, Chunma K and Ching W (2006), Boundary value method for solving trasient solution of Markovian queuing networks. Applied Mathematics and Computation, 172(2), 690-700.

[8] Chan RH, Lee ST and Sun HW( 2012), Boundary value methods for transient solutions of queueing networks with variant vacation policy, journal of Computational and Applied Mathematics, 236: 3948-3955.

[9] Czachorski T, Slaska TNP and Lipn FP (2009), Transient state analysis diffusion approximation as an alternative to Markov models, fluid flow approximation and simulation, Computers and Communication, 13-18.

[10] Grifiths JD, Leonenko GM and Williams JE (2013), The transient solution to M/Ek/1 queue. International Journal of Computer Applications, 61(21): 19-24.

[11] Gupta S (2011), A framework to span airport delay estimates using transient queuing models, Operations Research Center, Massachusetts Institute of Technology, Cambridge.

[12] Kaczynski WH, Leemis LM and Drew JH (2012), Transient queuing analysis, INFORMS journal on computing. 24(1), 10-28.

[13] Li M and Cheng J (2011), Transient state analysis of the shortest queueing system, International Conference on Artificial Intelligence, Management Science and Electronic Commerce AIMSEC.

[14] Monsellato A (2013), Transient analysis of a M/M/queue with discouragement and for the related embeded chain 3, http://arxiv.org/abs/1204.6618.

[15] Pearn WL and Chang YC (2004), Optimal management of N-policy M/Ek/1 queuing system with a removal service station, journal of computer and operation research, 31: 1001-10055.

[16] Radhika TSL and Deepika BLR (2014), State space approach for solving transient queuing systems, Transy/vanian Journal of Mathematics and Mechanics 6(1), 53-57.

[17] Singla N and Garg PC (2014), Transient and numerical solution of a feedback queueing system with correlated departures, American Journal of Numerical Analysis 2(1), 20-28.

[18] Vaderna P and Elteto T (2006), Transient analysis of a queuing system with matrix-geometric methods, Next Generation Teletraffic and Wired/Wireless Advanced Networking Lecture Notes in Computer Science 4003, 21-33, http://link.springer.com/chapter/10.1007

[19] Yang F and Liu J (2010), Transient analysis of general queue systems via simulation based transferred function modeling. Proceedings of the 2010 Winter Simulation Conference, USA. $1110-1122$.

[20] Yue D, Li C and Yue W (2006), Nonlinear dynamic and system theory. 6(3), 295-308.

[21] Zeng M, Cheng W and Guo P (2014), A transient queuing model for analyzing and optimizing gate, congestion of railway container terminals. Hindawi Publishing Corporation Mathematical Problems in Engineering, 1-13. 